\section{Computer selection of verbal research materials}

\section{ROBERT H. LOGIE \\ MRC Applied Psychology Unit, Cambridge, United Kingdom}

Most studies of verbal learning, memory, and perception require the selection of stimulus material the characteristics of which are chosen to study, or to control for, one or more of the attributes on which verbal material may vary. For example, in a study of the effects of written word frequency on recall, the researcher would not only be required to select words on the basis of frequency, but also to control for their length in number of letters, degree of imageability, and perhaps even the age at which the words are typically acquired.

A number of reports have been published that provide norm ratings for a wide variety of word attributes, (e.g., Gilhooly \& Logie, 1980; Paivio, Yuille, \& Madigan, 1968; Toglia \& Battig, 1978). However, the process of selecting materials from such sets of norms can be extremely time consuming and is greatly eased if much of the selection is carried out by computer. Coltheart (1981) and others are developing just such a facility, using sets of norms from a variety of sources with a total data base of 98,538 words, and providing a potentially invaluable resource. However, not all attributes are given for each word, and since differing sets of norms are involved, the measures for each attribute are not wholly consistent. In addition, at present, the size of the data base and the wide variety of parameters available require the researcher to submit requests for verbal material by post, in a specialized, although relatively straightforward, access language. This entails a necessary delay between submission of jobs and receipt of the sets of stimuli. Furthermore, the initial run on the system may not be wholly successful, and the job may have to be resubmitted. One solution to this would be on-line, interactive computer selection with a program and data base that are sufficiently small to be implemented on the computing facilities readily available in most psychology departments. The program to be described aims to provide such a facility.

Data Base. The program WORDSAMP uses as its data base the Gilhooly and Logie (1980) measures of age of acquisition, imagery, concreteness, familiarity, and ambiguity for 1,944 words. Thorndike and Lorge (1944) frequencies are also included for all words, all of which can be used as nouns. The program also allows sampling on the basis of length in number of letters, and on the

Thanks are due to Allan Maclean and Alan Baddeley at the APU, for comments on an earlier draft of this paper, and to Steve Platt, also at the APU, for computing advice. The author's mailing address is: MRC Applied Psychology Unit, 15 Chaucer Road, Cambridge, United Kingdom. occurrence of specified letter strings. This is a more modest data base than that used by Coltheart (1981). However, it contains consistently derived measures from a relatively homogeneous population and should be sufficient for most applications. It also allows for the flexibility of interactive sampling.

User Interface. The program is designed to be interactive with the user at each stage of the selection process. When the program is run, the user is given a brief description of the program and of the word attributes available from the Gilhooly and Logie (1980) data base. The instructions explain that the selection is performed by an iterative process, initially based on the parameters of the first word attribute specified. The sample selected then serves as the data base for selection on the basis of the second attribute chosen by the user. Values may be specified for as many of the available attributes as may be required for a particular application. However, users are warned that over-specification may result in very small (and peculiar) sets of words. The program indicates the number of words in the sample at completion of each stage of the selection.

Users are then asked if they require a purely random sample of words. If this is required, the user indicates the sample size, and the sample is written to a specified file name.

If a purely random sample is not required, a menu is presented, indicating the word attributes on which selection may be based and a range of values for each. Attribute selection is accomplished by typing the singleletter codes associated with each attribute. The program then requests maximum and minimum values for the first attribute chosen and selects all the words in the data base the values of which for that attribute fall within the specified range. The user is then informed of the size of the sample selected and is requested to specify the maximum and minimum values for the second attribute chosen. This process continues until sampling is complete for all attributes selected. The final sample size is given and the user may: (1) write the entire final sample to a permanent file; (2) make a smaller random selection from the final sample; (3) start again from the beginning; or (4) exit from the program. If the user chooses to write the sample to a file, a file name is requested, and the sample of words, together with attribute values and the mean and standard deviation for each set of values, is written to the specified file. The program then returns to the four choices outlined above.

On exiting from the program, the sample may be examined as it is for any other text file, or may be sent to a printer.

Program Description. The program consists of a main routine and a number of subroutines that handle the selection procedures. The MAIN routine controls program flow and contains sets of instructions for the user. One 
group of subroutines consists of one of each of the following available word attributes: imagery (IMAGER), age of acquisition (AGEAC), familiarity (FAMIL), concreteness (CONC), ambiguity (AMBIG), and frequency (FREQ). These serve to set up the range of values that the user selects for that particular attribute. These each in turn call the subroutine SAMPLE, which accesses the data base and selects words that fall within the range of values specified.

Two additional subroutines set up the search parameters and carry out the sampling for length (LENGTH) and for specific letter strings (STRING). Two further subroutines (RANDSA and RAND) are designed for random sampling from the total data base or from samples already selected on the basis of one or more attributes. Subroutine WRITE prompts the user for a file name to which the final sample of words is written, together with the attribute values for each word. It also calculates the mean and standard deviation for the attribute values in the sample.

The data base is contained in a separate, direct-access file that is opened and closed by the MAIN routine.

Language and System Requirements. The program is written in FORTRAN and may be compiled under FORTRAN 4 Plus or FORTRAN 77, and has been implemented on a DEC PDP-11/60 with RSX-11M operating system. However, the FORTRAN source file is available, and, with minor modifications, the program could be implemented on any machine that supports these versions of FORTRAN.

The sampling routine relies on the data-base file to be in direct-access format in order to minimize memory requirements. The program requires $30 \mathrm{~K}$ of memory to run.

Availability. The program and data base are available for use on an 8-in. floppy disk, which should accompany any requests for copies. Please state whether RSX-11 or RT-11 format is required.

\section{REFERENCES}

Coltheart, M. (February 1981). MRC Psycholinguistic Database User Manual: Version l. Department of Psychology, Birkbeck College, Malet Street, London.

Gilhooly, K. J., \& Logie, R. H. (1980). Age-of-acquisition, imagery, concreteness, familiarity, and ambiguity measures for 1,944 words. Behavior Research Methods \& Instrumentation, $12,395-427$

Paivio, A., Yuille, J. C., \& Madigan, S. (1968). Concreteness, imagery and meaningfulness values for 925 words. Journal of Experimental Psychology Monographs, 76(3, Part. 2).

Thorndike, E. L., \& LoRge, I. (1944). The Teachers word book of 30,000 words. New York: Teacher's College, Columbia University Press.

Toglia, M. P., \& Battig, W. F. (1978). Handbook of semantic word norms. New York: Erlbaum.

(Manuscript accepted for publication January 30, 1984.) 